\title{
AN EXTENSION OF E. G. STRAUS' PERFECT LATIN 3-CUBE OF ORDER 7
}

\author{
JOSEPH ARKIN
}

In memory of Ernst Straus

In a letter dated January, 1976 E. G. Straus sent this author a detailed construction of a $7 \times 7 \times 7$ perfect magic cube written to base 7 with digits 000 to 666 . In this construction he superimposed 3 Latin cubes of order 7 (a Latin 3-cube of order 7) to get what may be the lowest possible order of a perfect cube. Before Straus' construction the smallest known perfect cube was of order 8 . The Straus cube is perfect in the following way: the sum (2331) of the elements in each minor diagonal and in each pan-diagonal is equal to the sum of the elements of a row in each of the 2 directions in each of the respective squares (layers) that make up this perfect Latin 3-cube of order 7. The sum (2331) of the elements of a row in each direction of the cube is equal to the sum of the elements in each of the 4 major diagonals and the sum on all the diagonals of the cube is the same (namely 2331 ). The construction of the cube is based on the 3 orthogonal cubes

$$
\begin{gathered}
A_{i j k}^{(2)}=x_{i}+2 x_{j}-3 x_{k}, \quad A_{i j k}^{(-2)}=x_{i}-2 x_{j}-3 x_{k}, \\
A_{i j k}^{(3)}=x_{i}+3 x_{j}+2 x_{k}
\end{gathered}
$$

where $\left(x_{1}, \ldots, x_{7}\right)=(0,1, \ldots, y)$ and arithmetic is $(\bmod 7)$.

In this paper we superimpose 6 orthogonal Latin cubes of order 7 where each ordered triple $(000,001, \ldots, 666)$ occurs in every one of the 6 possible positions to form 20 separate Straus cubes.

A Latin square of order $n$ is an $n \times n$ square in which each of the numbers $0,1, \ldots, n-1$ occurs exactly once in each row and exactly once in each column. For example

$\begin{array}{lll}01 & 012 & 0123 \\ 10 & 120 & 1230 \\ & 201 & 2301 \\ & & 3012\end{array}$

are Latin squares of order 2, 3, 4, respectively. Two Latin squares of order $n$ are orthogonal, if when one is superimposed on the other, every ordered pair $00,01, \ldots, n-1 n-1$ occurs. Thus
012
012
$00 \quad 11$
22
120 and 201
superimpose to
1220
01
201
120
$21 \quad 02 \quad 10$ 
and therefore are orthogonal squares of order 3. A set of Latin squares of order $n$ is orthogonal if every two of them are orthogonal. As an example the $4 \times 4$ square of triples

$\begin{array}{llll}000 & 111 & 222 & 333 \\ 123 & 032 & 301 & 210 \\ 231 & 320 & 013 & 102 \\ 312 & 203 & 130 & 021\end{array}$

represents three mutually orthogonal squares of order 4 since each of the 16 pairs $00,01, \ldots, 33$ occurs in each of the three possible positions among the 16 triples.

We can generalize all these concepts to $n \times n \times n$ cubes and cubes of higher dimensions. A Latin cube of order $n$ is an $n \times n \times n$ cube ( $n$ rows, $n$ columns and $n$ files) in which the numbers $0,1, \ldots, n-1$ are entered so that each number occurs exactly once in each row, column and file. If we list the cube in terms of the $n$ squares of order $n$ which form its different levels we can list the cubes

\begin{tabular}{|c|c|c|c|}
\hline 01 & 10 & 012 & 120 \\
\hline 10 & 01 and & 120 & $\begin{array}{lll}2 & 0 & 1\end{array}$ \\
\hline
\end{tabular}

as Latin cubes of order 2 and 3, respectively.

Orthogonality of Latin cubes is the following relation among three Latin cubes: three Latin cubes of order $n$ are orthogonal if, when superimposed, each ordered triple $000,001, \ldots, n-1 n-1 n-1$ will occur. For example the pair of $3 \times 3$ Latin squares

$\begin{array}{lll}00 & 11 & 22 \\ 12 & 20 & 01 \\ 21 & 02 & 10\end{array}$

leads to the four $3 \times 3 \times 3$ cubes

\begin{tabular}{|c|c|c|c|}
\hline A: & $\begin{array}{lll}0 & 1 & 2 \\
1 & 2 & 0 \\
2 & 0 & 1\end{array}$ & $\begin{array}{lll}1 & 2 & 0 \\
2 & 0 & 1 \\
0 & 1 & 2\end{array}$ & $\begin{array}{lll}2 & 0 & 1 \\
0 & 1 & 2 \\
1 & 2 & 0\end{array}$ \\
\hline B: & $\begin{array}{lll}0 & 1 & 2 \\
2 & 0 & 1 \\
1 & 2 & 0\end{array}$ & $\begin{array}{lll}1 & 2 & 0 \\
0 & 1 & 2 \\
2 & 0 & 1\end{array}$ & $\begin{array}{lll}2 & 0 & 1 \\
1 & 2 & 0 \\
0 & 1 & 2\end{array}$ \\
\hline C: & $\begin{array}{lll}0 & 2 & 1 \\
2 & 1 & 0 \\
1 & 0 & 2\end{array}$ & $\begin{array}{lll}1 & 0 & 2 \\
0 & 2 & 1 \\
2 & 1 & 0\end{array}$ & $\begin{array}{lll}2 & 1 & 0 \\
1 & 0 & 2 \\
0 & 2 & 1\end{array}$ \\
\hline D: & $\begin{array}{lll}0 & 2 & 1 \\
1 & 0 & 2 \\
2 & 1 & 0\end{array}$ & $\begin{array}{lll}1 & 0 & 2 \\
2 & 1 & 0 \\
0 & 2 & 1\end{array}$ & $\begin{array}{lll}2 & 1 & 0 \\
0 & 2 & 1 \\
1 & 0 & 2\end{array}$ \\
\hline
\end{tabular}


Superimposed these lead to a cube of quadruples in three levels with I over II over III,

$\mathrm{ABCD}$ :

$\begin{array}{ccccccccc}0000 & 1122 & 2211 & 1111 & 2200 & 0022 & 2222 & 0011 & 1100 \\ 1221 & 2010 & 0102 & 2002 & 0121 & 1210 & 0110 & 1202 & 2021 \\ 2112 & 0201 & 1020 & 0220 & 1012 & 2101 & 1001 & 2120 & 0212 \\ & \text { I } & & & \text { II } & & & \text { III } & \end{array}$

where each ordered triple occurs in every one of the four possible positions in the quadruples.

Note. We define a cube of triples (say $\mathrm{ABC}$ ) where each ordered triple occurs in some order in the 27 cells of the three levels (I over II over III) of the cube $\mathrm{ABC}$ as a Latin 3-cube of order 3.

In this paper we superimpose the construction for 6 orthogonal Latin cubes of order 7 where each ordered triple $(000,001, \ldots, 666)$ occurs in every one of the 6 possible positions to form 20 separate Straus cubes is as follows.

\begin{tabular}{llllllll}
363330 & 525524 & 050011 & 212205 & 444462 & 606656 & 131143 & I. \\
416016 & 641203 & 103460 & 335654 & 560141 & 022335 & 254522 & \\
532465 & 064652 & 226146 & 451333 & 613520 & 145014 & 300201 & \\
655144 & 110331 & 342525 & 504012 & 036206 & 261463 & 423650 & \\
001523 & 233010 & 465204 & 620461 & 152655 & 314142 & 546336 & \\
124202 & 356466 & 511653 & 043140 & 205334 & 430521 & 662015 & \\
240651 & 402145 & 634332 & 166526 & 321013 & 553200 & 015464 & \\
\hline 006065 & 231252 & 463446 & 625633 & 150120 & 312314 & 544501 & II. \\
122444 & 354631 & 516125 & 041312 & 203506 & 435063 & 660250 & \\
245123 & 400310 & 632504 & 164061 & 326255 & 551442 & 013636 & \\
361502 & 523066 & 055253 & 210440 & 442634 & 604121 & 136315 & \\
414251 & 646445 & 101632 & 333126 & 565313 & 020500 & 252064 & \\
530630 & 062124 & 224311 & 456505 & 611062 & 143256 & 305443 & \\
653316 & 115503 & 340060 & 502254 & 034441 & 266635 & 421122 & \\
\hline 412423 & 644610 & 106104 & 331361 & 563555 & 025042 & 250236 & III. \\
535102 & 060366 & 222553 & 454040 & 616234 & 141421 & 303615 & \\
651551 & 113045 & 345232 & 500426 & 032613 & 264100 & 426364 & \\
004230 & 236424 & 461611 & 623105 & 155362 & 310556 & 542043 & \\
120616 & 352103 & 514360 & 046554 & 201041 & 433235 & 665422 & \\
243365 & 405552 & 630046 & 162233 & 324420 & 556614 & 011101 & \\
366044 & 521231 & 053425 & 215612 & 440106 & 602363 & 134550 & \\
\hline 125151 & 350345 & 512532 & 044026 & 206213 & 431400 & 663664 & IV. \\
241530 & 403024 & 635211 & 160405 & 322662 & 554156 & 016343 & \\
364216 & 526403 & 051660 & 213154 & 445341 & 600535 & 132022 & \\
410665 & 642152 & 104346 & 336533 & 561020 & 023214 & 255401 & \\
533344 & 065531 & 220025 & 452212 & 614406 & 146663 & 301150 & \\
656023 & 111210 & 343404 & 505661 & 030155 & 262342 & 424536 & \\
002402 & 234666 & 466153 & 621340 & 153534 & 315021 & 540215 & \\
\hline & & & & & & & \\
& & & & & & \\
& & &
\end{tabular}




\begin{tabular}{llllllll}
531516 & 063003 & 225260 & 450454 & 612641 & 144135 & 306322 & V. \\
654265 & 116452 & 341646 & 503133 & 035320 & 260514 & 422001 & \\
000644 & 232131 & 464325 & 626512 & 151006 & 313263 & 545450 & \\
123323 & 355510 & 510004 & 042261 & 204455 & 436642 & 661136 & \\
246002 & 401266 & 633453 & 165640 & 320134 & 552321 & 014515 & \\
362451 & 524645 & 056132 & 211326 & 443513 & 605000 & 130264 & \\
415130 & 640324 & 102511 & 334005 & 566262 & 021456 & 253643 & \\
\hline 244244 & 406431 & 631625 & 163112 & 325306 & 550563 & 012050 & VI. \\
360623 & 522110 & 054304 & 216561 & 441055 & 603242 & 135436 & \\
413302 & 645566 & 100053 & 332240 & 564434 & 026621 & 251115 & \\
536051 & 061245 & 223432 & 455626 & 610113 & 142300 & 304564 & \\
652430 & 114624 & 346111 & 501305 & 033562 & 265056 & 420243 & \\
005116 & 230303 & 462560 & 624054 & 156241 & 311435 & 543622 & \\
121565 & 353052 & 515246 & 040433 & 202620 & 434114 & 666301 & \\
\hline 650602 & 112166 & 344353 & 506540 & 031034 & 263221 & 425415 & VII. \\
003351 & 235545 & 460032 & 622226 & 154413 & 316600 & 541164 & \\
126030 & 351224 & 513411 & 045605 & 200162 & 432356 & 664543 & \\
242416 & 404603 & 636160 & 161354 & 323541 & 555035 & 010222 & \\
365165 & 520352 & 052546 & 214033 & 446220 & 601414 & 133601 & \\
411544 & 643031 & 105225 & 330412 & 562606 & 024163 & 256350 & \\
534223 & 066410 & 221604 & 453161 & 615355 & 140542 & 302036 &
\end{tabular}

In the above construction each ordered triple (a Straus cube) occurs in every one of the six possible positions in the sectuples.

Note. The Straus cube is mentioned in The Scientific American, February 1976, p. 127.

Received May 30, 1984.

197 OLD NYACK TURNPIKE

SPRING VALLEY, NY 10977 